\title{
Evaluation of the Clinical and Imaging Findings of Breast Examinations in a Tertiary Facility in Ghana
}

\author{
Emmanuel Kobina Mesi Edzie ${ }^{D},{ }^{1,2}$ Klenam Dzefi-Tettey ${ }^{(D}{ }^{3}$ Philip Narteh Gorleku ${ }^{1}{ }^{1}$ \\ Adu Tutu Amankwa $\left(\mathbb{D}^{4},{ }^{4}\right.$ Eric Aidoo $\left(\mathbb{D},{ }^{5}\right.$ Kwasi Agyen-Mensah $\left(\mathbb{D},{ }^{2,6}\right.$

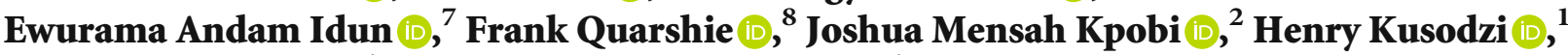 \\ Richard Ato Edzie $\mathbb{D}^{1}{ }^{1}$ and Abdul Raman Asemah $\mathbb{D}^{1}$
}

${ }^{1}$ Department of Medical Imaging, School of Medical Sciences, College of Health and Allied Sciences, University of Cape Coast, Cape Coast, Ghana

${ }^{2}$ Department of Radiology, Cape Coast Teaching Hospital, Cape Coast, Ghana

${ }^{3}$ Department of Radiology, Korle Bu Teaching Hospital, 1 Guggisberg Avenue, Accra, Ghana

${ }^{4}$ Department of Radiology, School of Medical Sciences, College of Health Sciences, Kwame Nkrumah University of Science and Technology, Kumasi, Ghana

${ }^{5}$ Department of Anatomy, School of Medical Sciences, College of Health and Allied Sciences, University of Cape Coast, Cape Coast, Ghana

${ }^{6}$ Department of Neurosurgery, School of Medical Sciences, College of Health and Allied Sciences, University of Cape Coast, Cape Coast, Ghana

${ }^{7}$ Department of Radiology, 37 Military Hospital, Neghelli Barracks Liberation Road 37, Accra, Ghana

${ }^{8}$ African Institute for Mathematical Sciences (AIMS), Summerhill Estates, East Legon Hills, Santoe, Accra, Ghana

Correspondence should be addressed to Emmanuel Kobina Mesi Edzie; emmanuel.edzie@ucc.edu.gh

Received 29 January 2021; Revised 8 July 2021; Accepted 9 July 2021; Published 20 July 2021

Academic Editor: Pranshu Sahgal

Copyright ( $) 2021$ Emmanuel Kobina Mesi Edzie et al. This is an open access article distributed under the Creative Commons Attribution License, which permits unrestricted use, distribution, and reproduction in any medium, provided the original work is properly cited.

Breast diseases have been one of the major battles the world has been fighting. In winning this fight, the role of medical imaging cannot be overlooked. Breast imaging reveals hidden lesions which aid physicians to give the appropriate diagnosis and definitive treatment, hence this study, to determine the clinical and imaging findings of breast examinations to document the radiologic features in our setting. This cross-sectional retrospective study reviewed the sociodemographics, imaging reports (mammography and ultrasonography with BI-RADS scores and their features), and the clinical data of 425 patients from September 2017 to September 2020 in the Cape Coast Teaching Hospital. 72 solid lesions with their histology reports were also reviewed. Data obtained were organized, coded, and analyzed using Statistical Package for Social Sciences (SPSS Inc., Chicago, IL, USA) version 20.0. The results obtained were presented in appropriate tables and charts. A chi-squared test was employed for associations and statistical significance was specified at $p \leq 0.05 .63 .29 \%$ of the patients were married, but only $18.59 \%$ had a positive family history of breast cancer. BI-RADS scores $1(57.46 \%)$ and $2(27.99 \%)$ were the most recurrent findings. The most common BI-RADS 2, 3, 4, and 5 imaging features were benign-looking axillary lymph nodes (66.33\%), well-defined solid masses (61.54\%), ill-defined solid masses (42.86\%), and ill-defined solid masses with suspicious-looking axillary lymph nodes $(100.00 \%)$, respectively. The most frequent indications were routine screening (49.18\%), mastalgia (26.59\%), and painless breast masses (19.77\%). There was significant association between duration of symptoms and breast cancer $(p$ value $=0.007)$. In conclusion, routine breast screening and mastalgia were the topmost indications for breast imaging. BI-RADS 1 and 2 were the commonest BI-RADS scores, and benign-looking axillary lymph nodes and simple cysts were the most frequent imaging features for BI-RADS 2 and ill-defined solid masses and suspicious-looking axillary lymph nodes for BI-RADS 4 and 5. 


\section{Introduction}

Breast-related diseases have become a topic of focus these days; one of such diseases is breast cancer. According to the World Health Organization (WHO), breast cancer is a leading cancer among women affecting about 2.1 million each year, causing the greatest number of cancer-related mortalities among women [1]. Breast hypertrophy, radial scars, breast cysts, fibroadenomas, intraductal papillomas, sclerosing adenosis, Phyllodes tumors and many more, are other breastrelated diseases [2]. The early stages of some of these breast conditions cause some pains whilst others do not. There is therefore the need for a frequent breast examination so as to aid early detection of such conditions, especially the ones that are not painful at the early stages [3].

There are forms of breast examinations; the popular one is the breast self-examination (BSE). The BSE is a known technique that an individual uses to examine his/her breast tissues for any change whether palpable or visual. It is often used as an early detection method for breast cancers/tumors [4]. The BSE technique was developed over 67 years ago from an idea proposed by a chapter of the American Cancer Society to a standard of recommendation of many health care professionals [5]. For economic and other reasons, BSE has been very important and easily accessible technique for people who could not access the clinical breast examination (CBE) which is usually done by the clinicians $[6,7]$.

Breast imaging is a subspecialty of diagnostic radiology. It generally refers to ultrasonography, mammography, and magnetic resonance imaging (MRI) of the breast. [8, 9]. Other modalities that may be used include positron emission tomography (PET), scintimammography, electrical impedance-based imaging, thermography, optical imaging, and computed tomography (CT) [10]. Breast imaging has so much importance; it mainly helps to define an injury to living tissue and to screen the remainder of the breast for secondary lesions. In general, breast imaging is done before a biopsy because the artifact from the biopsy can interfere with the interpretation of the study [11]. It also helps to identify and characterize breast masses and calcifications. Differentiation of cystic masses from solid masses are done through ultrasonography which is the most available imaging modality in Ghana $[12,13]$.

The American College of Radiology has advanced a system named BI-RADS which stands for Breast Imaging Reporting and Data System. The BI-RADS was developed for reporting mammogram results using a common language, for standardization and for patients' follow-ups. The radiologist assigns a single-digit BI-RADS score (ranging from 0 to 5 ) when the report of a person's mammogram is created [13]. The details of the BI-RADS scores are shown in (Table 1).

There is also BI-RADS 6 representing already diagnosed breast cancers, but this is not a routine category in the BIRADS lexicon. At this stage, it is advisable for the doctors to discuss the treatment plan with the patients. Currently, BI-RADS scores have been used in ultrasound and MRI imaging of the breasts $[13,14]$.

In a report by the World Cancer Research Fund, it was stated that the first four countries that recorded the highest cases of breast cancer in 2018 were all European countries, namely, Belgium, followed by Luxembourg, the Netherlands, and France (metropolitan), but they have comparatively lower mortality rates [15]. For instance, the mortality rates for breast cancer in Western and Northern Europe were 15.5 per 100,000 and 14.1 per 100,000 , respectively, in 2018, even though they had high incidence rates/100,000 (92.6 and 90.1 , respectively) $[16,17]$. In sharp contrast, the majority of women who die from breast cancer, live in low- and middle-income countries most of which are in Africa [16, 17]. A recent study reported that death rates of breast cancer in Western and Northern Africa were 17.8 per 100,000 and 18.4 per 100,000, respectively, which was comparatively higher even though they had lower incidence rates/100,000 (37.3 and 48.4, respectively) [16, 18].

In a study by Ghartey et al., nearly $70 \%$ of women diagnosed with breast cancer in Ghana were in advanced stages of the disease, mainly due to low awareness, with a resultant limited treatment success and a high mortality rate (15.2 per 100,000) $[19,20]$. Another study by Brakohiapa et al. also stated that educational programs on early breast cancer detection had a positive impact on the target population $[21,22]$, thereby encouraging frequent breast imaging and check-ups throughout the year and not only in the month of October which is the breast cancer awareness month. This study sought to examine the clinical and imaging findings of breast examinations in order to document the most recurrent radiologic features in our setting. This would help in our collective fight against breast-related diseases which has a relatively high mortality rate in Africa. The specific objectives of the study were as follows:

(i) To ascertain the sociodemographics of patients and commonest indications for breast imaging

(ii) To know the distribution of BI-RADS scores and imaging features contributing to the BIRADS scores

(iii) To determine whether there is an association between the family history of breast cancer and cancer cases

(iv) To determine whether there is an association between duration of symptoms, against cases of breast cancer and marital status

\section{Materials and Methods}

2.1. Study Site and Design. This was a cross-sectional retrospective study that reviewed the imaging records, sociodemographics, and clinical data of all patients $(n=425)$, who underwent breast imaging in the Radiology Department of the Cape Coast Teaching Hospital (CCTH) from September 2017 to September 2020. CCTH is the largest public health facility in the Central Region of Ghana. It offers tertiary and subspecialty services (including medical imaging services) to the inhabitants of the region and beyond. It is currently the training center for the University of Cape Coast medical school and a lead research institution in the region.

2.2. Data Collection. The data obtained included the age, marital status, history/clinical indication, a family history of 
TABLE 1: BI-RADS score categories.

\begin{tabular}{lcc}
\hline BI-RADS score & Category & Detail \\
\hline BI-RADS 0 & Incomplete & Mammogram study is not yet complete. \\
BI-RADS 1 & Negative & Mammogram was negative (no cancer). \\
BI-RADS 2 & Benign & Mammogram was normal (no cancer) but other findings (such as cysts) are described in the report. \\
BI-RADS 3 & Probably benign & Mammogram is probably normal/benign. Chances of breast cancer approximately 2\%. \\
BI-RADS 4 & Suspicious & Mammogram is probably malignant. Chances of breast cancer approximately 23\%-34\%. \\
BI-RADS 5 & High malignancy & Highly suspicious for malignancy with 95\% chance of breast cancer. \\
\hline
\end{tabular}

${ }^{*}$ Footnote: American College of Radiology. Breast Imaging Reporting and Data System (BIRADS) [14].

breast cancer, duration of symptoms, and the BI-RADS scores from breast imaging reports over the study period. All the patients within the study period were consecutively selected with no exclusions made. These imaging (mammography and ultrasonography) reports were done by two radiologists of ten years of experience in breast imaging reporting. The mammograms were acquired using FUJIFILM AMULET Digital Mammography System, Model FDR-2000AWS, 2012, manufactured by FUJIFILM Corporation (Tokyo, Japan), and the complementary ultrasonography was also performed using Toshiba Diagnostic Ultrasound System, Model Applio 300/TUS-A300, 2013, with a 7.5 MHz linear probe, manufactured by Toshiba Medical Systems (Otawara, Tochigi, Japan). The reviewed breast imaging reports that identified solid masses had their biopsy reports retrieved and categorized as negative for cancer and positive for cancer. The family history of breast cancer was categorized as "positive" and "negative"; the duration of symptoms which was initially a discrete variable, was also categorized as " $<1$ month," "1-12 months," "13-24 months," "25-36 months," "37-48 months," and ">48 months." Similar categorization was done for the marital status ("single" or "married"). The age of the participants was also categorized as "31-40 years," "41-50 years," "51-60 years," "61-70 years," and ">70 years," to help to know the distribution of breast cancer cases among the age groups. BI-RADS scores for all the patients were obtained from the combined reports of mammography and complementary ultrasound which is the routine practice in CCTH. BI-RADS scores from BI-RADS 2 to BI-RADS 5 for each patient were further analyzed to obtain the definite features that contributed to the BI-RADS categorization, as BI-RADS 1 is indicative of normal/negative breast findings. The BI-RADS scores from the reviewed reports were used to determine the proportions of "bilateral breast abnormality," "bilateral normal breasts," and "unilateral breast abnormality" in our setting. The laterality of the breast abnormality was further determined.

2.3. Statistical Analysis. The data obtained (age, marital status, clinical indication for breast imaging, a family history of breast cancer, duration of symptoms, the BI-RADS scores, and various lesions identified under each BI-RADS score categorization) were organized, coded, and analyzed using Statistical Package for Social Sciences (SPSS Inc., Chicago, IL, USA) version 20.0. The results obtained were presented in appropriate tables and charts using LibreOffice Calc
TABLE 2: Demographics, family history of breast cancer, duration of symptoms, and the distribution of the BI-RADS scores.

\begin{tabular}{lc}
\hline Items & Counts (\%) \\
\hline Age & 32 \\
Minimum & 85 \\
Maximum & $52.43(9.97)$ \\
Mean (SD) & \\
Age groups & $31(7.29 \%)$ \\
31-40 years & $198(46.59 \%)$ \\
$41-50$ years & $109(25.65 \%)$ \\
$51-60$ years & $60(14.12 \%)$ \\
$61-70$ years & $27(6.35 \%)$ \\
$>70$ years & \\
Marital status & $156(36.71 \%)$ \\
Single & $269(63.29 \%)$ \\
Married & \\
Family history of breast cancer & $79(18.59 \%)$ \\
Positive & $346(81.41 \%)$ \\
Negative & \\
Duration of symptoms & $33(15.28 \%)$ \\
$<1$ month & $141(65.28 \%)$ \\
$1-12$ months & $13(6.02 \%)$ \\
13-24 months & $15(6.94 \%)$ \\
25-36 months & $4(1.85 \%)$ \\
37-48 months & $6(1.63 \%)$ \\
Overall average duration of symptoms & \\
BI-RADS scores & $312(57.69 \%)$ \\
BI-RADS 2 & \\
BI-RADS 3 & \\
BI-RADS 4 & \\
\hline
\end{tabular}

(version 1:6.1.5-3+deb10u6 developed by The Document Foundation). A chi-squared test was employed to examine for any possible association among the categorized variables above (duration of symptoms, marital status, breast cancer cases, and family history of breast cancer). Statistical significance for this study was specified at $p \leq 0.05$. 
TABLE 3: Indications.

\begin{tabular}{|c|c|c|c|c|}
\hline Indications & Both breasts & Right breast & Left breast & Total (\%) \\
\hline Screening & - & - & - & $209(49.18 \%)$ \\
\hline Mastalgia & $99(71.22 \%)$ & $2(4.76 \%)$ & $12(27.27 \%)$ & $113(26.59 \%)$ \\
\hline Painless breast mass & $23(16.55 \%)$ & $35(83.33 \%)$ & $26(59.09 \%)$ & $84(19.77 \%)$ \\
\hline Painful breast mass & - & $2(4.76 \%)$ & $1(2.27 \%)$ & $3(0.71 \%)$ \\
\hline Discharge from breast & $3(2.16 \%)$ & $1(2.38 \%)$ & $2(4.55 \%)$ & $6(1.41 \%)$ \\
\hline Breast tenderness & $10(7.19 \%)$ & - & - & $10(2.35 \%)$ \\
\hline Heaviness in the breast & $1(0.72 \%)$ & - & $1(2.27 \%)$ & $2(0.47 \%)$ \\
\hline Burning sensation & $3(2.16 \%)$ & - & - & $3(0.71 \%)$ \\
\hline Breast swelling & - & $1(2.38 \%)$ & $2(4.55 \%)$ & $3(0.71 \%)$ \\
\hline Ulcerated breast lump & - & $1(2.38 \%)$ & - & $1(0.24 \%)$ \\
\hline
\end{tabular}

2.4. Ethical Consideration. Ethical clearance number (CCTHERC/EC/2020/093) for this study was obtained from the Ethical Review Committee of CCTH. Informed consent was not required for this study as this was a retrospective study but anonymity and confidentiality were ensured. This study conformed to the 1975 Declaration of Helsinki.

\section{Results}

Patients included in this study were only females as no male was seen for breast imaging over the study period. A total of 425 patients included in this study came for breast imaging (mammography and complementary ultrasonography) which were used for the BI-RADS categorization. The mean age of the participants was 52.43years $(\mathrm{SD}=9.97)$ with the age range of 32-85years. The rest of the sociodemographics of the patients are shown in (Table 2). Only 79 (18.59\%) of the reviewed patients had a positive family history of breast cancer. The BI-RADS distribution for this study is shown in (Table 2). Most of the patients presented with symptoms that had lasted between 1 to 12 months at the time of imaging and the duration of symptoms prior to breast imaging for the rest of the patients are shown in (Table 2).

The most common indications for breast imaging apart from screening (49.18\%) were breast pain accounting for $26.35 \%$ of the study population followed by painless breast masses (19.77\%). Also, the most frequent indication for symptomatic bilateral breast presentation was pain (71.22\%) whilst unilateral presentation was painless breast masses, $83.33 \%$ and $59.09 \%$ for the right and left breasts, respectively. The other indications are shown in (Table 3 ).

The majority of the patients 221 (52\%) had negative findings in both breasts (BI-RADS 1). 113 (26.59\%) had bilateral abnormal breasts and 91 (21.41\%) had unilateral abnormal breast, meaning they had BI-RADS 1 on one breast and any other BI-RADS score on the other breast apart from BI-RADS 1 (Figure 1). The commonest features for unilateral and bilateral breast imaging abnormalities were benign-looking axillary lymph nodes constituting $31.30 \%$ and $50.00 \%$, respectively. The second most recurrent abnormal feature for a unilateral breast was well-defined solid masses (18.32\%) followed by illdefined solid masses (14.50\%). Bilateral breast abnormalities featured ill-defined solid masses (10.64\%) as the second most

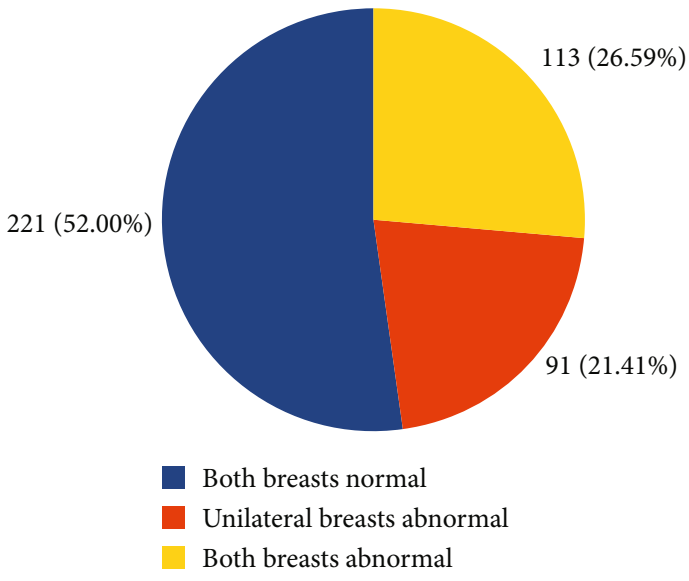

Figure 1: Chart representing the number of patients that had both breasts normal, unilateral breast abnormal, and both breast abnormal.

prevalent finding, followed by macrocalcifications (5.85\%) and well-defined cystic masses (5.85\%). The rest of the abnormal features are shown in (Table 4).

The imaging features that contributed to the abnormal BI-RADS scores (BI-RADS 2-5) are shown in (Table 4).

The right breast was more commonly affected than the left breast in cases of unilateral breast abnormality in this study (Figure 2).

Only benign imaging findings $(n=63)$ were seen in 55 patients out of the 209 patients who had breast imaging for routine screening. Out of the 425 patients included in the study, there were 72 solid breast masses out of which 42 were histologically cancerous. The distribution of the cancer patients is shown in Table 5, and distribution of cancer among the age groups is also shown in (Figure 3).

The majority of the patients that had cancerous solid masses $(57.1 \%)$ in this study significantly reported their symptoms from 1 to 12 months. There was no association between breast cancer and family history. The other associations are shown in (Table 6).

\section{Discussion}

Majority of the patients (92.61\%) who underwent breast imaging were above 40 years (Table 2 ). The modal age group 
TABLE 4: Abnormal BI-RADS scores and their associated imaging findings.

\begin{tabular}{|c|c|c|c|c|c|c|}
\hline Findings & BI-RADS 2 & BI-RADS 3 & BI-RADS 4 & BI-RADS 5 & $\begin{array}{c}\text { Unilateral } \\
\text { abnormality }\end{array}$ & $\begin{array}{c}\text { Bilateral } \\
\text { abnormality }\end{array}$ \\
\hline $\begin{array}{l}\text { Benign-looking axillary lymph } \\
\text { nodes }\end{array}$ & $132(66.33 \%)$ & $2(5.13 \%)$ & $1(1.43 \%)$ & - & $41(31.30 \%)$ & $94(50.00 \%)$ \\
\hline Acute mastitis & $11(5.53 \%)$ & - & - & - & $4(3.05 \%)$ & $7(3.72 \%)$ \\
\hline Macrocalcifications & $14(7.04 \%)$ & $4(10.26 \%)$ & $3(4.29 \%)$ & - & $10(7.63 \%)$ & $11(5.85 \%)$ \\
\hline Dilated ducts & $6(3.02 \%)$ & $3(7.69 \%)$ & $1(1.43 \%)$ & - & - & $9(4.79 \%)$ \\
\hline $\begin{array}{l}\text { Benign-looking intramammary } \\
\text { lymph nodes }\end{array}$ & $9(4.52 \%)$ & - & $1(1.43 \%)$ & - & $2(1.53 \%)$ & $8(4.26 \%)$ \\
\hline Lipoma & $1(0.50 \%)$ & - & - & - & $1(0.76 \%)$ & - \\
\hline Subcutaneous lesions & $2(1.01 \%)$ & - & - & - & - & $2(1.06 \%)$ \\
\hline Well-defined cystic masses & $17(8.54 \%)$ & $1(2.56 \%)$ & $2(2.86 \%)$ & - & $9(6.87 \%)$ & $11(5.85 \%)$ \\
\hline Periareolar skin thickening & $2(1.01 \%)$ & - & - & - & $2(1.53 \%)$ & - \\
\hline Abscess collection & $5(2.51 \%)$ & $1(2.56 \%)$ & - & - & $5(3.82 \%)$ & $1(0.53 \%)$ \\
\hline Well-defined solid masses & - & $24(61.54 \%)$ & $9(12.86 \%)$ & - & $24(18.32 \%)$ & $9(4.79 \%)$ \\
\hline Perilesional edema & - & - & $5(7.14 \%)$ & - & $2(1.53 \%)$ & $3(1.60 \%)$ \\
\hline Ill-defined solid mass & - & $3(7.69 \%)$ & $30(42.86 \%)$ & $6(50.00 \%)$ & $19(14.50)$ & $20(10.64 \%)$ \\
\hline Microcalcifications & - & $1(2.56 \%)$ & $8(11.43 \%)$ & - & $6(4.58 \%)$ & $3(1.60 \%)$ \\
\hline $\begin{array}{l}\text { Suspicious-looking axillary lymph } \\
\text { nodes }\end{array}$ & - & - & $10(14.29 \%)$ & $6(50.00 \%)$ & $6(4.58 \%)$ & $10(5.32 \%)$ \\
\hline
\end{tabular}

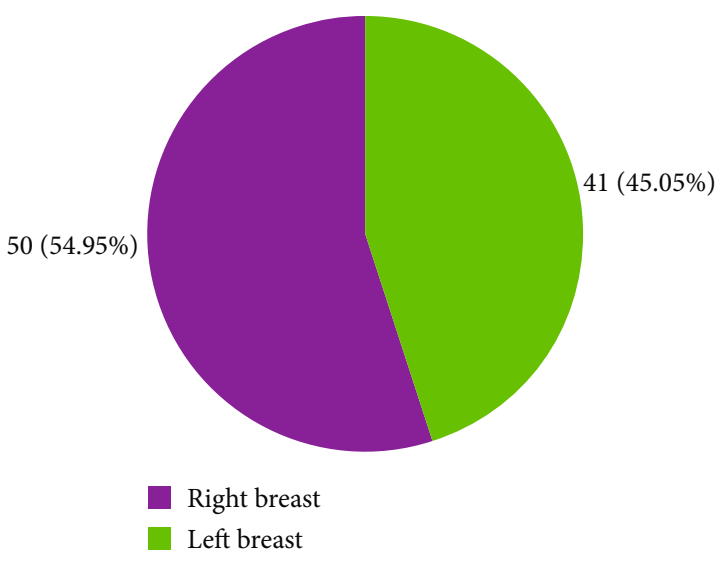

Figure 2: Abnormal unilateral breast.

was 41-50years, which is also the recommended age group for breast cancer screening [23]. This finding is therefore encouraging since it indicates that practitioners are requesting for breast imaging in line with recommended guidelines. The number of patients after the modal age group decreased with increasing age, which may be due to the relatively low life expectancy of 64.85 years for Ghanaian females [24]. The majority of the patients in this study were married $(63.29 \%)$, but there was no significant association ( $p$ value $=0.162$ ) between the marital status of the patients and duration of symptoms (Table 6).

A number of studies have shown that family history is one of the significant risk factors of breast cancer $[25,26]$. However, in our study, the majority $(73.8 \%)$ of the patients, with radiologically and histologically confirmed breast cancer, had no family history of breast cancer. This finding was not statistically significant $(p$ value $=0.511)$ (Table 6). Similar results have also been reported by $\mathrm{WHO}$ and other published articles. The WHO in March 2021 stated that the majority of women who develop breast cancer have no known family history of the disease [27]. In a study by Liu et al., only 10$15 \%$ of all breast cancer cases are associated with family history, with as high as about $85 \%$ having no association [28]. Other studies have also reported similar findings [29, 30]. This may be due to other factors such as oral contraceptive use, cigarette smoking, and menopause [31]. Another factor may be the relatively low number $(n=42)$ of patients with breast cancer in this study. The average duration of symptoms of the 42 patients with breast cancer in this study was $18.95 \pm 18.72$ months. This contrasts with a study in Nigeria by Ibrahim et al., which reported an average duration of symptoms of $12.12 \pm 5.18$ months which is relatively and comparatively lower than that of those in our study setting [32]. This may be due to the high level of spirituality, religion, and superstition assigned to the causes of many health issues. This affirms with studies in Ghana, which found the reversal of negative dreams, anointing women, laying of hands, revelation, and prayers as spiritual interventions by their spiritual leaders and pastors $[33,34]$. We found a significant association between duration of symptoms and cancerous solid masses or breast cancer cases $(p$ value $=0.007)$ (Table 6). The most frequent BI-RADS scores were BI-RADS 1 $(57.46 \%)$ and BI-RADS 2 (27.99\%), which is similar to what was found by Mahoney et al. [35].

Apart from routine screening, mastalgia (26.59\%) was the most common indication in this study (Table 3). Other studies have found similar results $[36,37]$. This may be due to the fact that pain perception is likely to be reported faster than any other symptom [38, 39]. Barton et al. reported breast pain as the most prevalent indication in their study 
TABLE 5: The distribution of imaging findings among the patients with abnormal BI-RADS scores who reported for routine screening and distribution of cancer patients.

\begin{tabular}{lcc}
\hline Imaging findings & Counts & Percentage \\
\hline Benign-looking axillary lymph nodes & 50 & $79.37 \%$ \\
Well-defined cystic mass & 1 & $1.59 \%$ \\
Acute mastitis & 2 & $3.17 \%$ \\
Macrocalcifications & 6 & $9.52 \%$ \\
Benign-looking intramammary lymph nodes & 4 & $6.35 \%$ \\
Distribution of cancer patients $(n=42)$ & & $7.14 \%$ \\
Bilateral & 3 & $92.86 \%$ \\
Unilateral & 39 & $53.85 \%$ \\
Right breast & 21 & $46.15 \%$ \\
Left breast & 18 \\
Average duration of symptoms & $18.95 \pm 18.72$ months \\
\hline
\end{tabular}

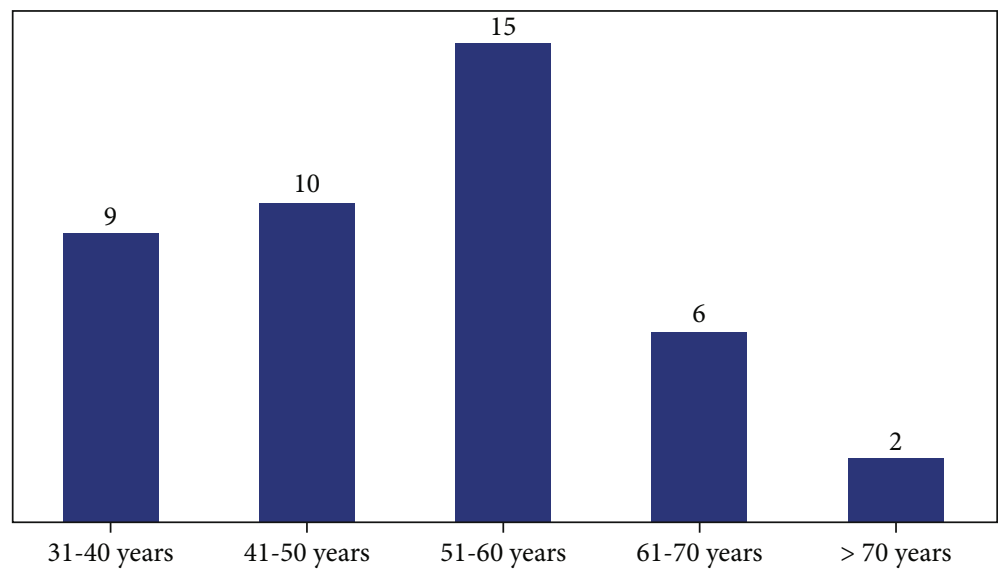

FIGURE 3: Distribution of cancer among the age groups.

TABLE 6: Cross-tabulation variables.

Cross-tabulation of duration of symptoms against solid masses and marital status

Duration of symptoms (in months)

\begin{tabular}{|c|c|c|c|c|c|c|c|c|}
\hline & $<1$ & $1-12$ & $13-24$ & $25-36$ & $37-48$ & $>48$ & $\chi^{-2}$ & $p$ value \\
\hline \multicolumn{9}{|l|}{ Solid masses } \\
\hline Positive for cancer & - & $24(57.1 \%)$ & $6(14.3 \%)$ & $7(16.7 \%)$ & $3(7.1 \%)$ & $2(4.8 \%)$ & 15.910 & \multirow{2}{*}{$0.007^{*}$} \\
\hline Negative for cancer & $1(3.3 \%)$ & $6(20.0 \%)$ & $6(20.0 \%)$ & $8(26.7 \%)$ & $1(3.3 \%)$ & $8(26.7 \%)$ & 15.910 & \\
\hline \multicolumn{9}{|l|}{ Marital status } \\
\hline Single & $10(13.0 \%)$ & $45(58.4 \%)$ & $8(10.4 \%)$ & $8(10.4 \%)$ & $1(1.3 \%)$ & $5(6.5 \%)$ & 7.897 & 0.162 \\
\hline Married & $23(16.5 \%)$ & $96(69.1 \%)$ & $5(3.6 \%)$ & $7(5.0 \%)$ & $3(2.2 \%)$ & $5(3.6 \%)$ & 7.897 & 0.162 \\
\hline
\end{tabular}

Cross-tabulation of family history of breast cancer against breast cancer cases

Family history of breast cancer

$$
\text { Positive Negative }
$$

\section{Solid masses}

\begin{tabular}{|c|c|c|c|c|}
\hline Positive for cancer & $11(26.2 \%)$ & $31(73.8 \%)$ & 0.432 & 0.511 \\
\hline Negative for cancer & $10(33.3 \%)$ & $20(66.7 \%)$ & & \\
\hline
\end{tabular}

*Statistically significant. 
population, also corroborated by other studies in Ghana [21, $40,41]$. We also found that pain in the left breast (85.71\%) was more common than pain in the right breast (14.29\%) which similarly had been reported by Clegg-Lamptey et al. They reported a much lower percentage of left breast pain $(52.41 \%)$ and a comparatively higher percentage of right breast pain $(47.59 \%)$ in their study [42]. This breast pain disparity was difficult to explain from this study, but it may be due to breast asymmetry also reported by other studies. [43, 44]. We found that the number of breasts with unilateral and bilateral cancerous solid masses after histology were $39(92.86 \%)$ and $3(7.14 \%)$, respectively, and also supported by Setz-Pels et al., who reported $97.8 \%$ (1766) and $2.2 \%(40)$ occurrences of unilateral and bilateral cancer, respectively [45]. Other studies also found similar results $[46,47]$.

Our current study found that all the imaging features for the BI-RADS 2 were benign features, and benign-looking axillary lymph nodes (66.33\%) were the most frequent, followed by breast cysts ( $8.54 \%)$. This finding is contrary to what was reported by Taylor et al., who found breast cysts as the most recurrent [48]. This may be because Taylor et al. did not include axillary lymph nodes as a BI-RADS 2 feature. The most prevalent BI-RADS 3 imaging features $(61.54 \%)$ were well-defined solid masses and macrocalcifications $(10.26 \%)$, which is also contrary to what was reported by Taylor et al. The most common BI-RADS 4 and BIRADS 5 features were ill-defined solid masses constituting $42.86 \%$ and $50.00 \%$, respectively, also confirmed by Taylor et al. (Table 4).

Even though routine breast examination has been advised by the WHO and other studies, most women do not give it the necessary attention $[49,50]$. In a study to know the perception and screening behaviors among Korean women in Australia, it was found that only $16.9 \%$ paid special attention to screening their breasts monthly [51]. The situation is known to be worse in Africa as previous reports had showed that the high mortality rate is due to low participation in regular checkups evolving from low awareness which finally leads to late diagnosis and treatment $[52,53]$. It is therefore worth noting that 55 out of the 209 patients who reported for routine check-ups had benign imaging features (Table 5). Early detection of these imaging findings would help the patients take early treatment to avoid disease progression.

Generally, we found that the majority of the unilateral abnormalities were in the right breast (54.95\%) which is similar to the findings of Thomas et al., who reported $50.11 \%$ unilateral right breast abnormality [54]. The majority of the cancerous lesions were found in the right breast (53.85\%) (Table 5) which is contrary to what Sisti et al. reported, where $50.60 \%$ of the recorded cancer cases were in the left breast [55]. A study in Ghana by Der et al. reported the majority of women with breast cancer were below 61 years old similar to what we also found (Figure 3) unlike the developed countries where most of the cancer patients are below 50 years [56-58]. This supports the finding that most females in Europe develop breast cancer at an earlier age than those in Africa $[31,57]$.
4.1. Limitations. The histological reports were obtained for only solid masses. The histology for other benign-looking lesions on imaging were not obtained, and only the imaging features were used since the focus of this study was not on histo-pathology. The conclusion from this study may be limited by the relatively small sample size. The sizes and locations of mass lesions were not considered. These could be areas for further research in our setting.

\section{Conclusion}

Mastalgia was the most common indication apart from routine screening, and it occurred more commonly in the left breast. The BI-RADS scores that occurred most frequently in this study were BI-RADS 1 and 2, followed by BI-RADS 4 , 3, and finally, BI-RADS 5. The commonest BI-RADS 2 features were benign-looking axillary lymph nodes and well-defined cystic masses. The most frequent BI-RADS 3 imaging features were well-defined solid masses followed by macrocalcifications and those of BI-RADS 4 and BI-RADS 5 were ill-defined solid masses and suspicious-looking axillary lymph nodes. Significant majority of the patients with breast cancer reported relatively early. There was no significant association between family history and breast cancer in this study.

\section{Data Availability}

The data used to support the findings of this study may be obtained upon request to the Head of research, CCTH at ccthresearch@gmail.com.

\section{Conflicts of Interest}

The authors declare that they have no conflicts of interest.

\section{Authors' Contributions}

All the authors contributed substantially to the conception, design, implementation of this article, and to the write-up, revision, and approval of the manuscript for publication.

\section{Acknowledgments}

The management of CCTH and the staff of the radiology department of the CCTH are appreciated for their support in making this study successful.

\section{References}

[1] A. Omar, A. Bakr, and N. Ibrahim, "Female medical students' awareness, attitudes, and knowledge about early detection of breast cancer in Syrian Private University, Syria," Heliyon, vol. 6, no. 4, article e03819, 2020.

[2] S. G. Komen, Benign breast conditions, 2015, January 2021, https://www.komen.org/breast-cancer/factsstatistics/benignbreast-conditions/.

[3] M. Onstad and A. Stuckey, "Benign breast disorders," Obstetrics and Gynecology Clinics, vol. 40, no. 3, pp. 459-473, 2013. 
[4] J. Austoker, "Breast self examination," BMJ, vol. 326, no. 7379, pp. 1-2, 2003.

[5] S. C. Eggertsen and J. J. Bergman, "Breast self-examination: historical perspective and current progress," The Journal of family practice, vol. 16, no. 4, pp. 713-716, 1983, https:// psycnet.apa.org/record/1984-02096-001.

[6] T. C. Jacob and N. E. Penn, "The need and value of breast selfexamination," Journal of the National Medical Association, vol. 80, no. 7, pp. 777-787, 1988.

[7] R. Dadzi and A. Adam, "Assessment of knowledge and practice of breast self-examination among reproductive age women in Akatsi South district of Volta region of Ghana," PLoS One, vol. 14, no. 12, article e0226925, 2019.

[8] Z. S. Lima, M. R. Ebadi, G. Amjad, and L. Younesi, "Application of imaging technologies in breast cancer detection: a review article," Open Access Macedonian Journal of Medical Sciences, vol. 7, no. 5, pp. 838-848, 2019.

[9] Inside Radiology, Breast imaging, 2020, January 2021, https:// www.insideradiology.com.au/?s=breast+imaging.

[10] S. V. Sree, E. Y. Ng, R. U. Acharya, and O. Faust, "Breast imaging: a survey," World journal of clinical oncology, vol. 2, no. 4, pp. 171-178, 2011.

[11] B. N. Joe and E. A. Sickles, "The evolution of breast imaging: past to present," Radiology, vol. 273, no. 2S, pp. S23-S44, 2014.

[12] E. K. M. Edzie, K. Dzefi-Tettey, P. N. Gorleku et al., “Application of information and communication technology in radiological practices: a cross-sectional study among radiologists in Ghana," Journal of Global Health Reports, vol. 4, article e2020046, 2020.

[13] L. Levy, M. Suissa, J. F. Chiche, G. Teman, and B. Martin, "BIRADS ultrasonography," European journal of radiology, vol. 61, no. 2, pp. 202-211, 2007.

[14] L. Liberman and J. H. Menell, "Breast imaging reporting and data system (BI-RADS)," Radiologic Clinics, vol. 40, no. 3, pp. 409-430, 2002.

[15] World Cancer Research Fund, Breast cancer statistics-breast cancer is the most common cancer in women worldwide, 2018, January 2021, https://www.wcrf.org/dietandcancer/ cancer-trends/breast-cancer-statistics.

[16] F. Bray, J. Ferlay, I. Soerjomataram, R. L. Siegel, L. A. Torre, and A. Jemal, "Global cancer statistics 2018: GLOBOCAN estimates of incidence and mortality worldwide for 36 cancers in 185 countries," CA: a cancer journal for clinicians, vol. 68, no. 6, pp. 394-424, 2018.

[17] F. Sardanelli, H. S. Aase, M. Álvarez et al., "Position paper on screening for breast cancer by the European Society of Breast Imaging (EUSOBI) and 30 national breast radiology bodies from Austria, Belgium, Bosnia and Herzegovina, Bulgaria, Croatia, Czech Republic, Denmark, Estonia, Finland, France, Germany, Greece, Hungary, Iceland, Ireland, Italy, Israel, Lithuania, Moldova, The Netherlands, Norway, Poland, Portugal, Romania, Serbia, Slovakia, Spain, Sweden, Switzerland and Turkey," European radiology, vol. 27, no. 7, pp. 2737-2743, 2017.

[18] D. Adeloye, O. Y. Sowunmi, W. Jacobs et al., "Estimating the incidence of breast cancer in Africa: a systematic review and meta-analysis," Journal of global health, vol. 8, no. 1, 2018.

[19] F. Naku Ghartey Jnr, A. Anyanful, S. Eliason, S. Mohammed Adamu, and S. Debrah, "Pattern of breast cancer distribution in Ghana: a survey to enhance early detection, diagnosis, and treatment," International journal of breast cancer, vol. 2016, 9 pages, 2016.

[20] D. Gyau and J. Nonvignon, Economic burden of breast cancer in women: a study at the KorleBu teaching hospital, University of Ghana, 2016, http://197.255.68.203/handle/123456789/ 21625.

[21] E. K. Brakohiapa, G. E. Armah, J. N. Clegg-Lamptey, and W. O. Brakohiapa, "Pattern of breast diseases in Accra: review of mammography reports," Ghana medical journal, vol. 47, no. 3, pp. 101-106, 2013, https://www.ajol.info/index.php/ gmj/article/view/99639.

[22] A. B. Bonsu, B. P. Ncama, and K. O. Bonsu, "Breast cancer knowledge, beliefs, attitudes and screening efforts by microcommunity of advanced breast cancer patients in Ghana," International Journal of Africa Nursing Sciences, vol. 11, p. 100155, 2019.

[23] D. Ikeda and K. K. Miyake, Breast Imaging: The Requisites EBook, Elsevier Health Sciences, 2004.

[24] StatistaJanuary 2021, https://www.statista.com/statistics/ 970703/life-expectancy-at-birth-in-ghana-bygender/.

[25] G. A. Colditz, B. A. Rosner, and F. E. Speizer, "Risk factors for breast cancer according to family history of breast cancer," JNCI: Journal of the National Cancer Institute, vol. 88, no. 6, pp. 365-371, 1996.

[26] P. D. Pharoah, N. E. Day, S. Duffy, D. F. Easton, and B. A. Ponder, "Family history and the risk of breast cancer: a systematic review and meta-analysis," International journal of cancer, vol. 71, no. 5, pp. 800-809, 1997.

[27] World Health Organization (WHO), Breast cancer, 2021, July 2021, https://www.who.int/news-room/fact-sheets/detail/ breast-cancer.

[28] L. Liu, X. Hao, Z. Song, X. Zhi, S. Zhang, and J. Zhang, "Correlation between family history and characteristics of breast cancer," Scientific Reports, vol. 11, no. 1, p. 6360, 2021.

[29] G. Haber, N. U. Ahmed, and V. Pekovic, "Family history of cancer and its association with breast cancer risk perception and repeat mammography," American journal of public health, vol. 102, no. 12, pp. 2322-2329, 2012.

[30] H. R. Brewer, M. E. Jones, M. J. Schoemaker, A. Ashworth, and A. J. Swerdlow, "Family history and risk of breast cancer: an analysis accounting for family structure," Breast cancer research and treatment, vol. 165, no. 1, pp. 193-200, 2017.

[31] G. O. Abdulrahman and G. A. Rahman, "Epidemiology of breast cancer in Europe and Africa," Journal of cancer epidemiology, vol. 2012, 5 pages, 2012.

[32] N. A. Ibrahim and M. A. Oludara, "Socio-demographic factors and reasons associated with delay in breast cancer presentation: a study in Nigerian women," The Breast, vol. 21, no. 3, pp. 416-418, 2012.

[33] L. Aziato, P. N. Odai, and C. N. Omenyo, "Religious beliefs and practices in pregnancy and labour: an inductive qualitative study among post-partum women in Ghana," BMC pregnancy and childbirth, vol. 16, no. 1, p. 138, 2016.

[34] E. K. M. Edzie, K. Dzefi-Tettey, P. N. Gorleku et al., "Perception of Ghanaian Primigravidas undergoing their first antenatal ultrasonography in Cape Coast," Radiology Research and Practice, vol. 2020, 10 pages, 2020.

[35] M. C. Mahoney, C. Gatsonis, L. Hanna, W. B. DeMartini, and C. Lehman, "Positive predictive value of BI-RADS MR imaging," Radiology, vol. 264, no. 1, pp. 51-58, 2012. 
[36] M. Morrow, "The evaluation of common breast problems," American family physician, vol. 61, no. 8, pp. 2371-8, 2385, 2000, https://www.aafp.org/afp/2019/0415/p505.html.

[37] B. Salzman, S. Fleegle, and A. S. Tully, "Common breast problems," American Family Physician, vol. 86, no. 4, pp. 343-349, 2012, https://www.aafp.org/afp/2012/0815/p343.html.

[38] L. Garfinkel, L. Craig, and H. Seidman, “An appraisal of left and right breast cancer," Journal of the National Cancer Institute, vol. 23, no. 4, pp. 617-631, 1959.

[39] H. Tulinius, H. Sigvaldason, and G. Olafsdottir, "Left and right sided breast cancer," PathologyResearch and Practice, vol. 186, no. 1, pp. 92-94, 1990.

[40] M. B. Barton, J. G. Elmore, and S. W. Fletcher, "Breast symptoms among women enrolled in a health maintenance organization: frequency, evaluation, and outcome," Annals of internal medicine, vol. 130, no. 8, pp. 651-657, 1999.

[41] M. Boadu, E. K. Sosu, F. Hasford et al., "Mammography examination in Ghana: preliminary survey of patients' profiles," Journal of Applied Science and Technology, vol. 17, no. 1, pp. 87-92, 2012, https://www.ajol.info/index.php/jast/article/ view/75213.

[42] J. N. Clegg-Lamptey, C. Edusa, N. Ohene-Oti, and J. A. Tagoe, "Breast cancer risk in patients with breast pain in Accra, Ghana," East African Medical Journal, vol. 84, no. 5, pp. 215-218, 2007.

[43] D. Scutt, G. A. Lancaster, and J. T. Manning, "Breast asymmetry and predisposition to breast cancer," Breast cancer research, vol. 8, no. 2, p. R14, 2006.

[44] J. F. Waljee, E. S. Hu, L. A. Newman, and A. K. Alderman, "Predictors of breast asymmetry after breast-conserving operation for breast cancer," Journal of the American college of Surgeons, vol. 206, no. 2, pp. 274-280, 2008.

[45] W. Setz-Pels, L. E. Duijm, J. H. Groenewoud et al., "Patient and tumor characteristics of bilateral breast cancer at screening mammography in the Netherlands, a population-based study," Breast cancer research and treatment, vol. 129, no. 3, pp. 955961, 2011.

[46] H. Takahashi, K. Watanabe, M. Takahashi, K. Taguchi, F. Sasaki, and S. Todo, "The impact of bilateral breast cancer on the prognosis of breast cancer: a comparative study with unilateral breast cancer," Breast Cancer, vol. 12, no. 3, pp. 196-202, 2005.

[47] S. A. Narod, "Bilateral breast cancers," Nature Reviews Clinical Oncology, vol. 11, no. 3, pp. 157-166, 2014.

[48] K. Taylor, P. Britton, S. O'keeffe, and M. G. Wallis, "Quantification of the UK 5-point breast imaging classification and mapping to BI-RADS to facilitate comparison with international literature," The British journal of radiology, vol. 84, no. 1007, pp. 1005-1010, 2011.

[49] I. L. Koinberg, L. Holmberg, and B. Fridlund, "Breast cancer patients' satisfaction with a spontaneous system of check-up visits to a specialist nurse," Scandinavian journal of caring sciences, vol. 16, no. 3, pp. 209-215, 2002.

[50] R. S. Kerrison, H. Shukla, D. Cunningham, O. Oyebode, and E. Friedman, "Text-message reminders increase uptake of routine breast screening appointments: a randomised controlled trial in a hard-to-reach population," British journal of cancer, vol. 112, no. 6, pp. 1005-1010, 2015.

[51] C. Kwok, M. J. Lee, and C. F. Lee, "Breast cancer perceptions and screening behaviours among Korean women in Australia,"
Journal of immigrant and minority health, vol. 22, no. 1, pp. 126-133, 2020.

[52] C. P. Akuoko, E. Armah, T. Sarpong, D. Y. Quansah, I. Amankwaa, and D. Boateng, "Barriers to early presentation and diagnosis of breast cancer among African women living in sub-Saharan Africa," PLoS One, vol. 12, no. 2, article e0171024, 2017.

[53] S. Y. Opoku, M. Benwell, and J. Yarney, "Knowledge, attitudes, beliefs, behaviour and breast cancer screening practices in Ghana, West Africa," Pan African Medical Journal, vol. 11, no. 1, 2012.

[54] A. S. Thomas, K. M. Kidwell, J. K. Oppong et al., "Breast cancer in Ghana: demonstrating the need for population-based cancer registries in low- and middle-income countries," Journal of global oncology, vol. 3, no. 6, pp. 765-772, 2017.

[55] A. Sisti, M. T. Huayllani, D. Boczar et al., "Breast cancer in women: a descriptive analysis of the national cancer database," Acta Bio Medica: Atenei Parmensis, vol. 91, no. 2, pp. 332-341, 2020.

[56] E. M. Der, R. K. Gyasi, and E. K. Wiredu, "Prognostication of breast cancer in Ghanaian women receiving modified radical mastectomy: a retrospective histopathological study at KorleBu teaching hospital, Accra, Ghana," Archives of Breast Cancer, vol. 1, pp. 129-137, 2018.

[57] R. L. Bowen, S. W. Duffy, D. A. Ryan, I. R. Hart, and J. L. Jones, "Early onset of breast cancer in a group of British black women," British journal of cancer, vol. 98, no. 2, pp. 277$281,2008$.

[58] C. K. Gajalakshmi, V. Shanta, R. Swaminathan, R. Sankaranarayanan, and R. J. Black, "A population-based survival study on female breast cancer in Madras, India," British journal of cancer, vol. 75, no. 5, pp. 771-775, 1997. 